\title{
TiN-Ag as an antimicrobial and wear resistant coating
}

\author{
Iñigo Braceras ${ }^{\mathrm{a}, *}$, Marta Brizuela ${ }^{\mathrm{a}}$, Noelia Álvarez ${ }^{\mathrm{a}}$, Miguel Martínez Van Geeteruyen ${ }^{\mathrm{b}}$, \\ Ibon Azkona ${ }^{\mathrm{c}}$
}

a TECNALIA, Basque Research and Technology Alliance (BRTA), Mikeletegi Pasealekua 2, Donostia-San Sebastián, Spain

b Sumisan S.A., Amasorrain kalea 3, 20018 Donostia-San Sebastián, Spain.

${ }^{\mathrm{c}}$ Metal Estalki S.L., Polígono Ugaldeguren II, 48170 Zamudio, Spain

\section{A R T I C L E I N F O}

\section{Keywords:}

TiN-Ag

Antimicrobial

Silver release

Electrical contact resistance

Friction

Surgical tool

\begin{abstract}
A B S T R A C T
Nosocomial infections are a major clinical concern, posing great risks for patients and rising costs for health services providers. This work aims at developing a hard, wear resistant coating, whose antimicrobial properties shall prevent the transmission of infections.

TiN coatings deposited by Physical Vapour Deposition, PVD, with different Ag contents have been studied, especially in relation to the hardness and adhesion, their microstructure and morphology. The antimicrobial activity of the surfaces has been assessed against Staphylococcus epidermidis at different time frames, one of the most troublesome source of infections in trauma and orthopaedic surgeries. The electro-tribology properties of different silver contest have been studied. Finally, the coatings have been deposited on surgical acetabular reamers and wear resistance tests have been carried out against synthetic composite bone (simulating cortical and cancellous bone).

Results have shown a good coating adhesion on stainless steel (both quantitatively in the scratch tests and qualitatively in the tests against synthetic composite bone), while the hardness decreased with higher Ag percentages. Furthermore, coatings exhibited antimicrobial activity against S.epidermidis, limited silver release, a remarkable wear resistance (vs. uncoated surgical acetabular reamers), while the electrical contact resistance provided valuable information about the evolution of friction and the status of the coating. Therefore TiN-Ag coatings present promising features for reducing the risk of infections, monitoring and extending cutting edge life and quality, and thus limiting damage to living tissues, e.g. necrosis.
\end{abstract}

\section{Introduction}

With modern protocols, the incidence of infections associated to the placement of implants has become very low: e.g. estimated in $0.5-5 \%$ for total joint replacements, [1] but up to $0.5 \%$ to $15 \%$ in patients undergoing primary or revision joint arthroplasty when considering high risk and oncological cases, [2] and even reaching up to $30 \%$ in open fractures. [3,4] Thus, infections still cause a huge impact in terms of morbidity, mortality, and medical costs. For instance, direct hospital costs, related to the management of prosthetic joint infections has been reposted to range from approximately $€ 20,000$ to $€ 60,000$, while the long-term economic effect of a post-surgical infection after joint arthroplasty has been calculated to exceed $\$ 390,000$ per case. [2] In any case, surgical infections are associated with poor clinical outcomes, prolonged hospital admissions, and complex revision operations, which are associated with their own complications, including far greater mortalities. [5] The pathogenesis of peri-implant infections is conditioned by the fact that the critical dose of contaminating microorganisms to produce an infection is much lower with a foreign material at the surgical site. [4] As in other medical fields, prevention represents the main tool, which relies on, among others, the control of environmental and personnel contamination and the establishment of effective protocols of peri-operative antibiotic prophylaxis. [1,3]

However, it is at the biomaterial surface-surrounding living tissue interface where accidental contamination can first develop into colonization, subsequently into biofilm formation and the establishment of a clinically relevant infection. [5] This is especially true in the case of surgical tools. [6] One convenient way to interfere with the early phases of microbial adhesion consists on the modification of the chemistry or the morphology of the out-layer of the devices in contact with living tissue. This can be achieved applying antimicrobial coatings, e.g. using plasma-based technologies. [2,7-9]

\footnotetext{
* Corresponding author at: Mikeletegi Pasealekua 2, 20009 Donostia-San Sebastián, Spain.

E-mail address: inigo.braceras@tecnalia.com (I. Braceras).
} 


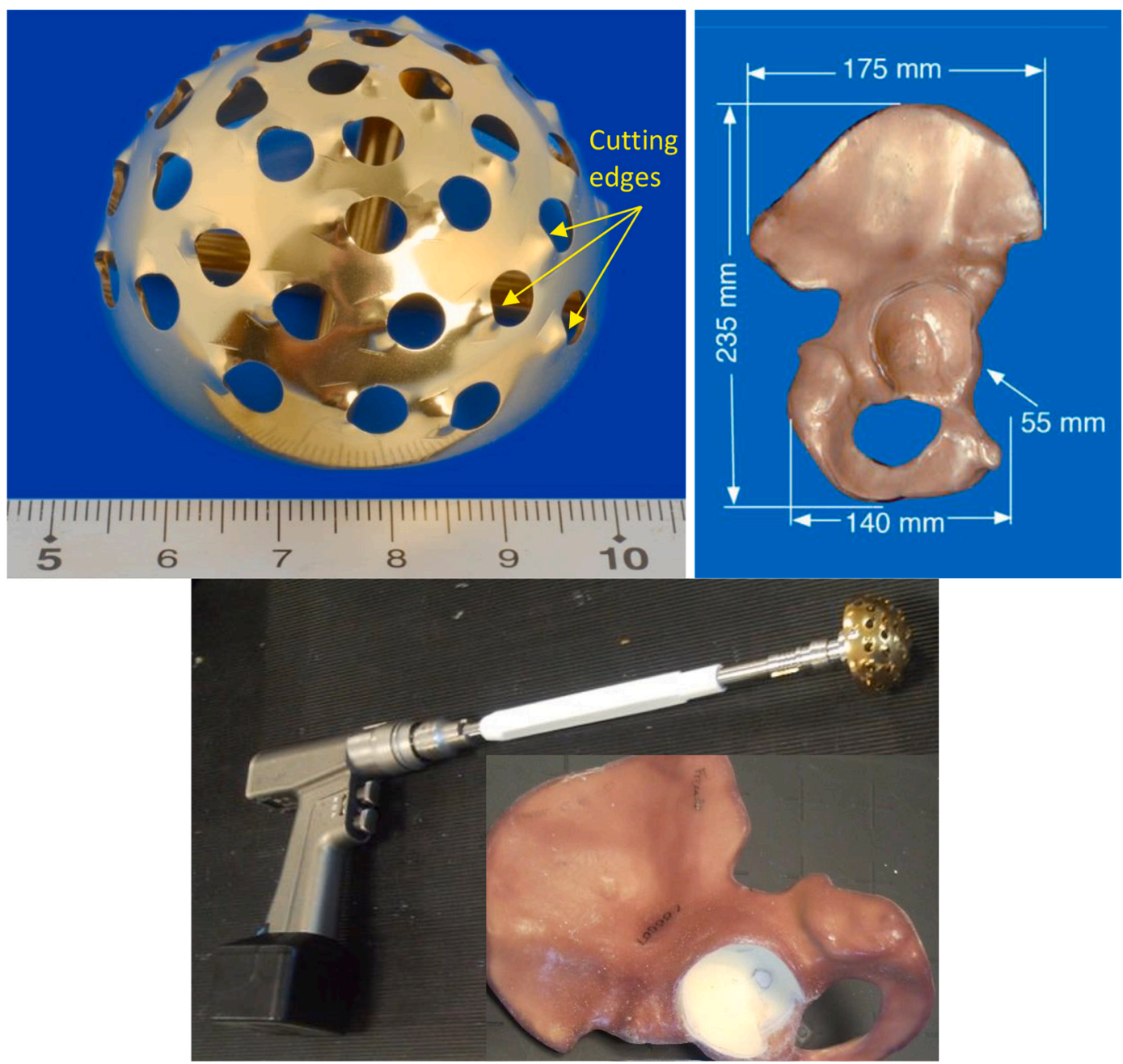

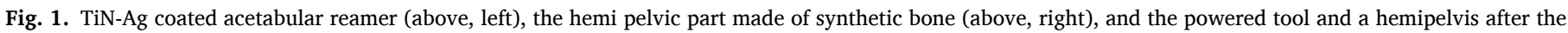
wear test (below).

However, in many applications, such coatings must also exhibit a good wear resistance, especially when interacting with hard living tissue such as bone. Physical Vapour Deposition (PVD) is an adequate technique to deposit hard wear resistant coatings, and is routinely being applied commercially on tools for many applications, e.g. machining, metal forming, etc. One way to add the antimicrobial function consist on doping a hard coating, such as the pioneering TiN coating, with metallic elements with known antimicrobial effect, e.g. Ag, Cu... [10-16] Specifically silver shows a strong bactericidal activity even at low concentrations, low toxicity, and most importantly abroad antibacterial spectrum against planktonic and sessile, Gram-positive and Gramnegative, and also multi drug-resistant bacteria. [17-20]

In this regard, this study has aimed at evaluating of the electrotribological and anti-microbial properties of PVD deposited TiN-Ag thin films with a wide range of silver concentrations.

\section{Material and Methods}

In this work, TiN-Ag coatings have been deposited on mirror polished AISI 420A stainless steel (50 $\mathrm{mm}$ in diameter and $5 \mathrm{~mm}$ thick), monocrystalline $\langle 100\rangle$ silicon samples (in various small sizes $<30 \times 10$ $\mathrm{mm})$, as well as glass slides $(75 \times 25 \times 1 \mathrm{~mm})$, by Physical Vapour Deposition (PVD). The surface finishing was in all substrates, coated and uncoated, $<$ Ra $0.05 \mu \mathrm{m}$. The chemical composition of AISI 420A, which is one of the materials of choice in surgical tools, is: C $0.20 \%$; Si $0.51 \%$; Mn 0.63\%; P 0.02\%; Cr 13.2\%; Ni 0.13\%.
The coatings were deposited by Magnetron Sputtering PVD (CemeCon CC800/8 PVD equipment) using (one or two) pure titanium and (one) silver solid targets $(200 \times 88 \mathrm{~mm})$; and nitrogen $(130 \mathrm{ml} / \mathrm{h})$, argon $(125 \mathrm{ml} / \mathrm{h})$ and krypton $(80 \mathrm{ml} / \mathrm{h})$ as process gasses, at a pressure of 900 $\mathrm{mPa}$. The main process variable consisted on the power applied on the silver target (from $0 \mathrm{~W}$ to $1000 \mathrm{~W}$ ), which is correlated with the amount of silver on the deposited coatings. The power applied on the titanium target was kept at $8000 \mathrm{~W}$, the DC bias voltage at $120 \mathrm{~V}$, the anode voltage at $115 \mathrm{~V}$, and the coating deposition process took place in either 6800 or $3400 \mathrm{~s}$, if one or two titanium targets were active respectively. The TiN coating deposited in the same conditions (i.e. without $\mathrm{Ag}$ ) was also included in the study.

Coating adherence on the steel substrates was characterized by scratch tests (ST103, VTT Technology, Finland). The scratch test method consists of applying a diamond tip on the surface with an increasing load, while the sample moves linearly, in this case increasing the load from 5 to $90 \mathrm{~N}$, at $10 \mathrm{~N} / \mathrm{mm}$. Afterwards the different kinds of damage done by the tip on the coating was analyzed at the optical microscopy and correlated with load applied. The coating ultra-microhardness was measured dynamically, increasing the load applied from 0.4 to $10 \mathrm{mN}$ (Fischerscope H100 dynamic microprobe apparatus).

The coating chemical composition was determined by Energy Dispersive Spectroscopy (EDS; OXFORD INCA-300, Witney, Oxon, UK), while the surface morphology was analyzed by Electron Scanning Microscopy (SEM; JEOL JSM-59110LV, Akishima City, Tokyo, Japan). The coating microstructure (on Si substrates) was also analyzed by glancing 
X-Ray Diffraction Spectroscopy (XRD; D8Advance XRD, Brucker, USA).

Concerning the antimicrobial activity, the complete description of modes of action remain unresolved, but it is known that silver deactivates enzymes by binding to thiol groups and inhibit the respiratory chain of bacteria. [9] In this study, the antimicrobial activity of the TiNAg surfaces was assessed in vitro against Staphylococcus epidermidis. Infections in early onset ( $<3$ months) and delayed onset (3 to 12-24 months) are thought to occur due to direct contamination at the time of surgery, with early onset being caused by more virulent microorganisms, such as $S$. aureus, and delayed/later onset being caused by a more indolent species such as $S$. epidermidis. [21,22] The testing procedure followed was based on the JIS Z2801 standard.

and was performed on three replica per coating variation (on glass slides), which were cleaned with ethanol. The procedure was in accordance with this standard but for one of the bacterium strains tested, S. epidermidis, and test durations. It consisted basically on inoculating $0.4 \mathrm{ml}$ of a bacterial concentration solution (approximately $0.25-110^{6}$ $\mathrm{cfu} / \mathrm{ml}$ ) onto each sample surface, covering them with a non-toxic nonbiocide polymer film, and incubating the samples for 6 and $24 \mathrm{~h}$ at $37^{\circ} \mathrm{C}$ and $100 \%$ of relative humidity. Afterwards the remaining bacteria were extracted with a stomacher (Masticator, Iul Instruments, Spain), the viable bacteria counted (cfu: number of colony forming units), and the value of antimicrobial activity, $\mathrm{R}$, calculated. Stomachers or Paddle Blenders are used in microbiology to extract and wash off microbes into solution through pressure and motion with electrically driven reciprocating paddles, which act on flexible plastic bags creating blending forces (i.e. the 'stomaching' action) without entering into contact with the processed samples. The number of viable bacteria was quantified following the agar plate culture method, as detailed by the standard. The value of antimicrobial activity was calculated as the percentage of reductions of viable bacteria on the coated materials with respect to uncoated materials after the respective test durations.

Additionally, the amount of silver released during the test was also quantified by Inductively Coupled Plasma Atomic Emission Spectroscopy, ICP-AES (VISTA-MPX, Varian). ICP-AES is a type of emission spectroscopy that uses the inductively coupled plasma to produce excited atoms and ions that emit electromagnetic radiation at wavelengths characteristic of a particular element, and where the intensity of the emissions are proportional to the concentrations of the elements within the sample.

Finally, the wear resistance of selected TiN-Ag coatings was assessed with two different methods. First, tribology tests were performed on a pin-on-disc configuration, at room temperature $\left(\sim 25^{\circ} \mathrm{C} ; 2 \mathrm{~N}\right.$ load; $50 \%$ relative humidity; $0.2 \mathrm{~m} / \mathrm{s}$; for approximately $24 \mathrm{~h}$ test duration), against $6 \mathrm{~mm}$ diameter WC-Co balls. Both the coefficient of friction (CoF) and the electrical contact resistance (ECR) were monitored along the tests. Tests were performed on a MT2/60/NI/HT Tribometer (Microtest, Spain), where the CoF is obtained through a strain gauge which measures the deflection of an elastic arm-holder, at the end of which a passive load is applied on the ball. The ECR was registered with a Keithley 2100 ultimeter measurement module, with the wiring fixed between a copper baseplate insulated electrically from the equipment on top of which the test sample is placed, and the ball holder, also electrically insulated from the rest of the equipment. Details of the equipment and test set up have been described elsewhere. [23,24] Medical devices related tribological conditions can vary enormously, specially concerning speed. In this case, the speed was selected as an approximation to the low average range of reamer speeds. [25,26] Wear tracks were analyzed by SEM and EDS.

Additionally, the coatings were deposited on acetabular reamers (Fig. 1, above left), made of AISI 420A stainless steel, which are typically used on the surgical procedures for the placement of the acetabular component of hip prostheses. The wear tests in this case were performed rotating (uni-directional motion) the coated and uncoated acetabular reamers against the acetabulum (hip socket) of hemipelvic parts (3 per reamer) made of synthetic bone, using the same powered tooling used in

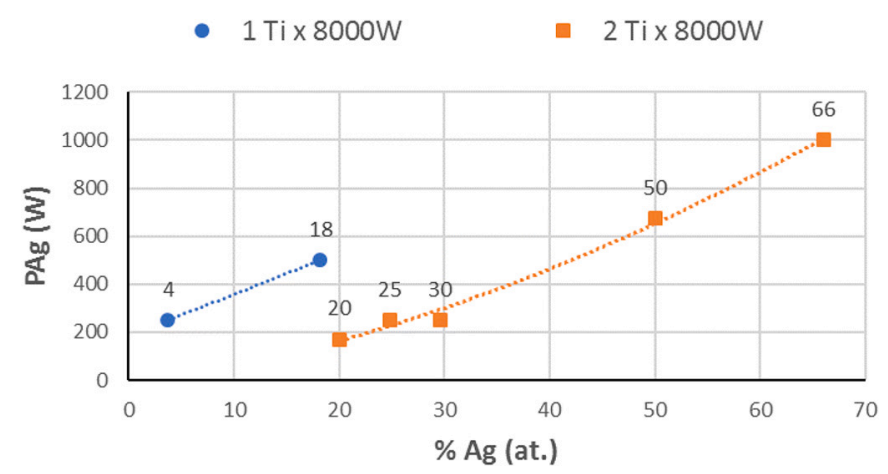

Fig. 2. Correlation between process parameters (power applied to the Ag target and use of one $(\boldsymbol{)})$ or two $(\square)$ targets of Ti) and the silver content in the deposited coatings.

surgical procedures and in dry conditions, till the cortical bone material had been completely removed (Fig. 1, below). The synthetic bone material has physical strength properties similar to real bone (Sawbones, Sweden, www.sawbones.com; Fig. 1, above right; short fiber filled epoxy as simulated cortical bone material and a cancellous foam core of $0.27 \mathrm{~g} / \mathrm{cm}^{3}$ ). The degree of wear on the cutting edges was measured at the Scanning Electron Microscope (SEM) (Fig. 9).

\section{Results and Discussion}

\subsection{Coating Composition, Hardness, Thickness and Adhesion}

TiN-Ag coatings were deposited using one or two titanium targets with a constant applied power ( $8000 \mathrm{~W}$ per target), and one silver target at different powers. The resulting coatings showed a wide variation in the silver content, ranging from $4 \%$ to $66 \%$ at. (Fig. 2), and colour, ranging from goldish to silver like surfaces. This is mainly based on the very high sputtering rate of $\mathrm{Ag}$, which means that small changes in power applied to the silver target lead to large variations in the silver content in the films. [27-29] The coatings have been referenced from this point as TiN-nnAg, where nn corresponds to the atomic silver percentage in the coating.

The silver content had a remarkable effect on the coating hardness (Fig. 3). As expected, the higher the silver content the softer the resulting coatings. The TiN coating exhibited a hardness of $\mathrm{HU}=31 \mathrm{GPa}$ and a modified Young Modulus of $233 \mathrm{GPa}$. With the addition of $20 \%$ silver into the coating, the hardness almost halved to $15.8 \mathrm{GPa}$, although it was still harder than the steel substrate, $8.9 \mathrm{GPa}$. However, with $30 \%$ silver, the hardness dropped to a value close to that of the substrate, i.e. 9.6 $\mathrm{GPa}$. And when the silver content raised to $66 \%$, the hardness dropped to $5.4 \mathrm{GPa}$, well below the steel substrate hardness. At lower silver contents, hardness was not significantly higher than that with $20 \%$ silver, certainly lower than the values reported by other authors for comparable silver contents. [13,14]

The coating thicknesses ranged from 1.7 to $7 \mu \mathrm{m}$ (Fig. 3). The thickest corresponded to TiN-66Ag and the thinnest to TiN-04Ag, i.e. the ones with the largest and smallest amount of silver. In all cases the indentation depth in the hardness measurement was less than $10 \%$ of the coating thickness, with the exception of ref. TiN-04Ag where it reached $13 \%$.

On the other hand, the coating adhesion was affected to a lesser extent by the silver content, which still caused a slight decrease. Qualitatively though, a fragile failure was observed in the TiN coating, while a ductile one occurred on TiN-66Ag (Fig. 4), which is expected in alloys with high silver contents, which present lower residual stresses. [15,23] 

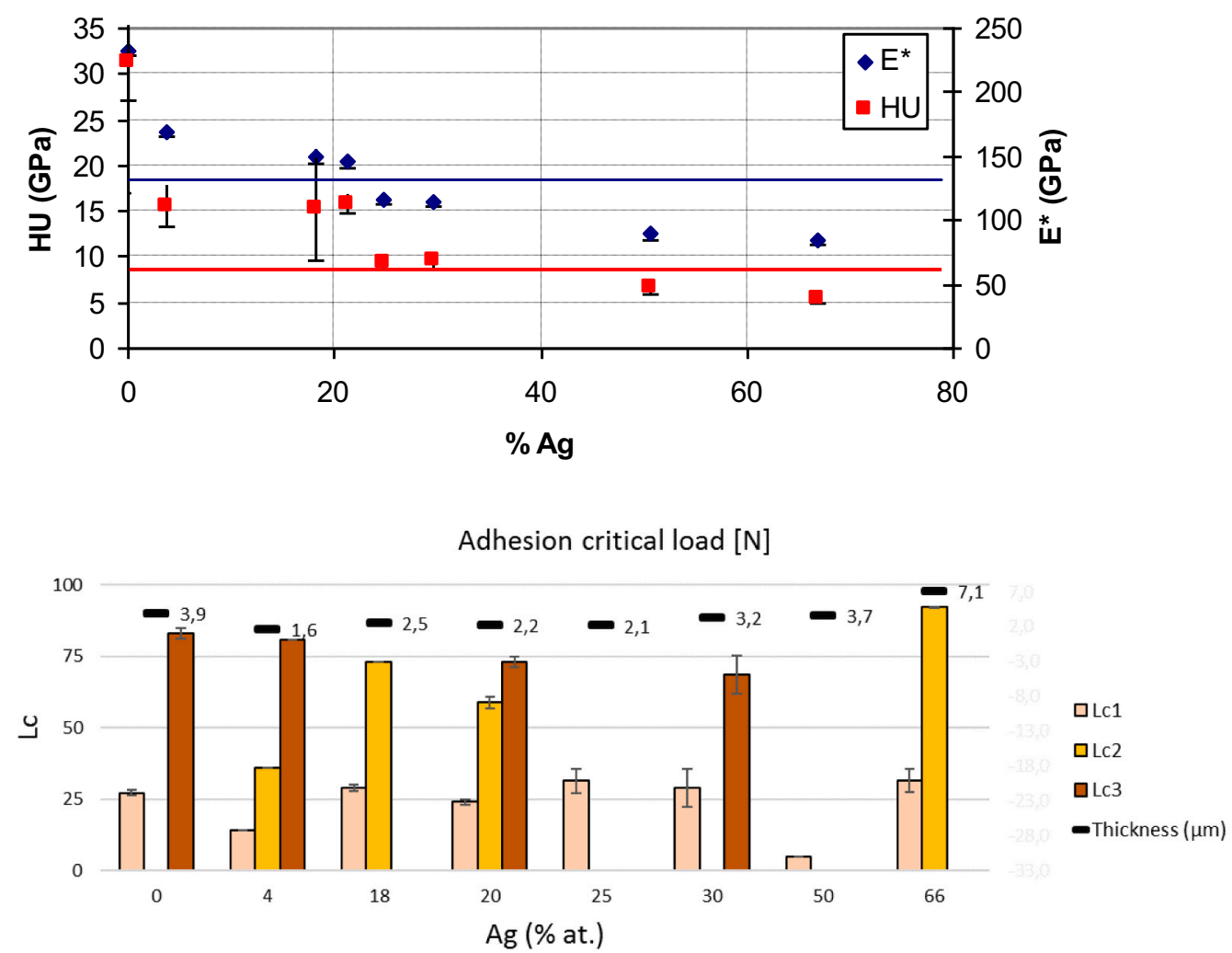

Fig. 3. Coating universal hardness, HU, the modified Young Modulus $\mathrm{E}^{*}$ (above; the red line indicates the HU of the stainless steel substrate, the blue line its $\mathrm{E}^{*}$ ), adhesion and thickness (below). (For interpretation of the references to colour in this figure legend, the reader is referred to the web version of this article.)

a)

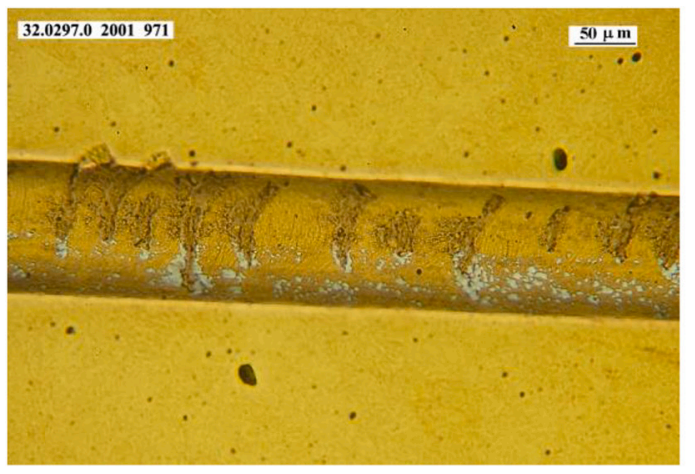

c)

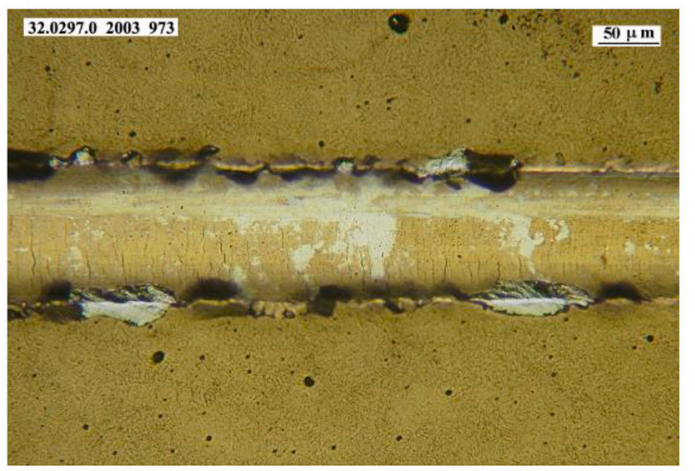

b)

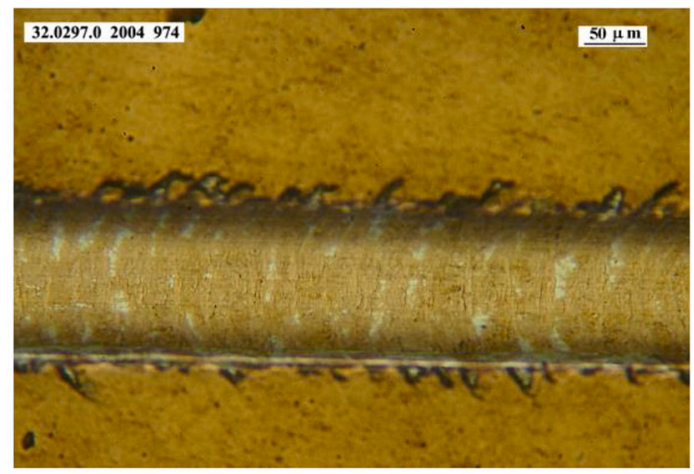

d)

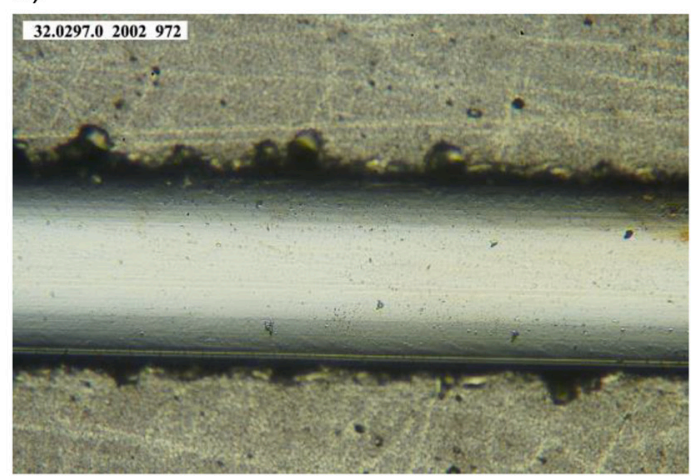

Fig. 4. Images of the scratch test tracks in coatings ref. a:TiN, b:TiN-20Ag, c:TiN-30Ag, d:TiN-66Ag. 

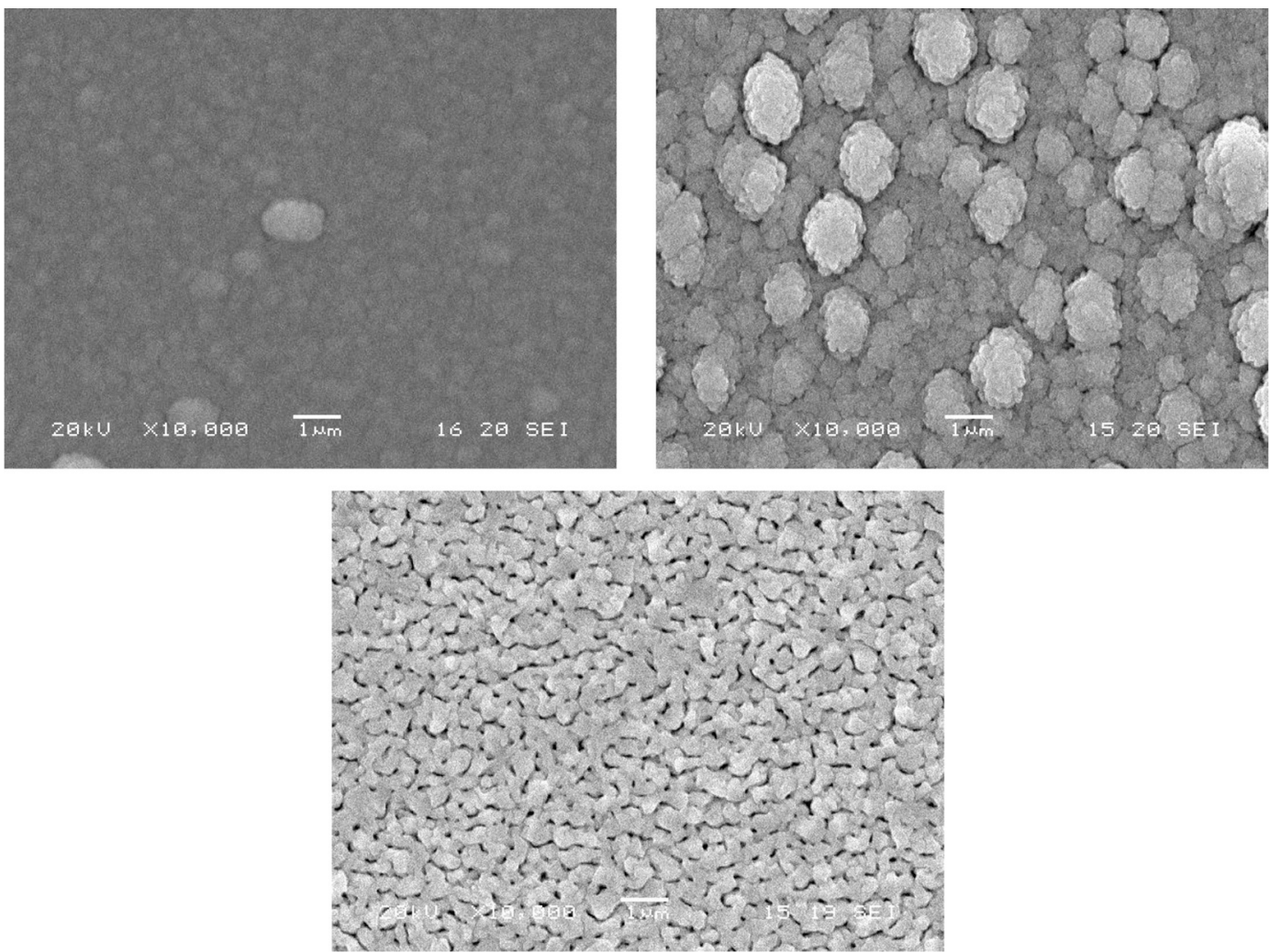

Fig. 5. SEM images of the coating surfaces of ref. TiN-20Ag (top, left), TiN-30Ag (top right) and TiN-66Ag (bottom).
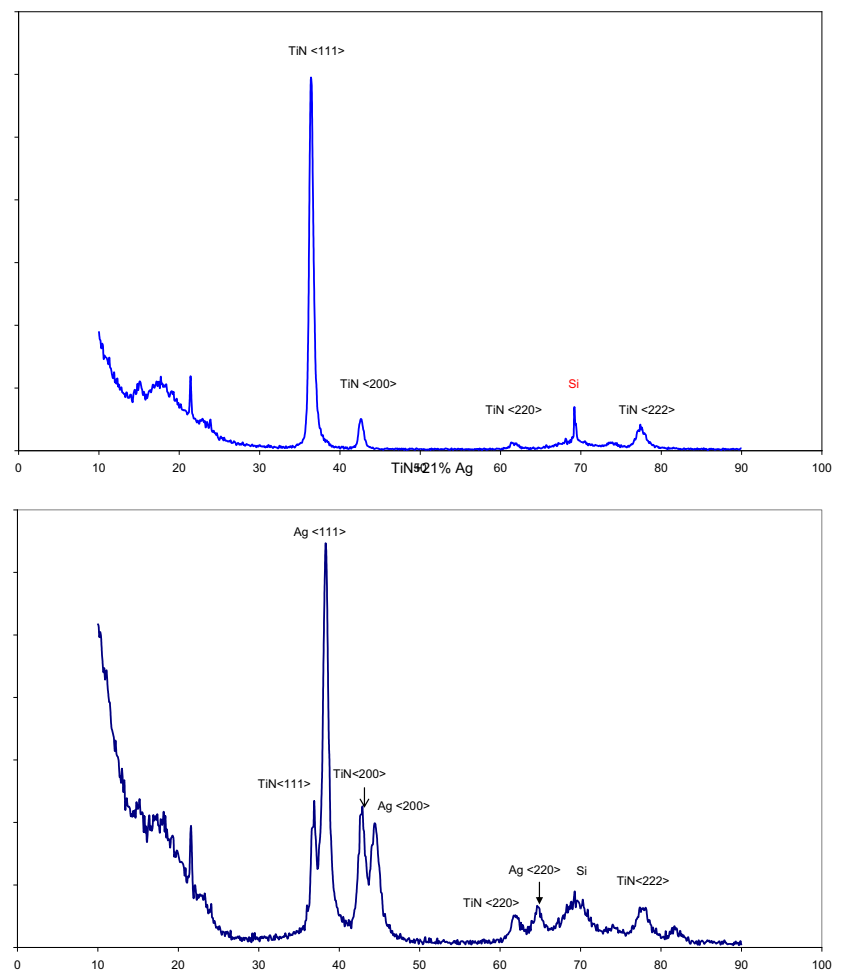

$\mathrm{TiN}+30 \% \mathrm{Ag}$
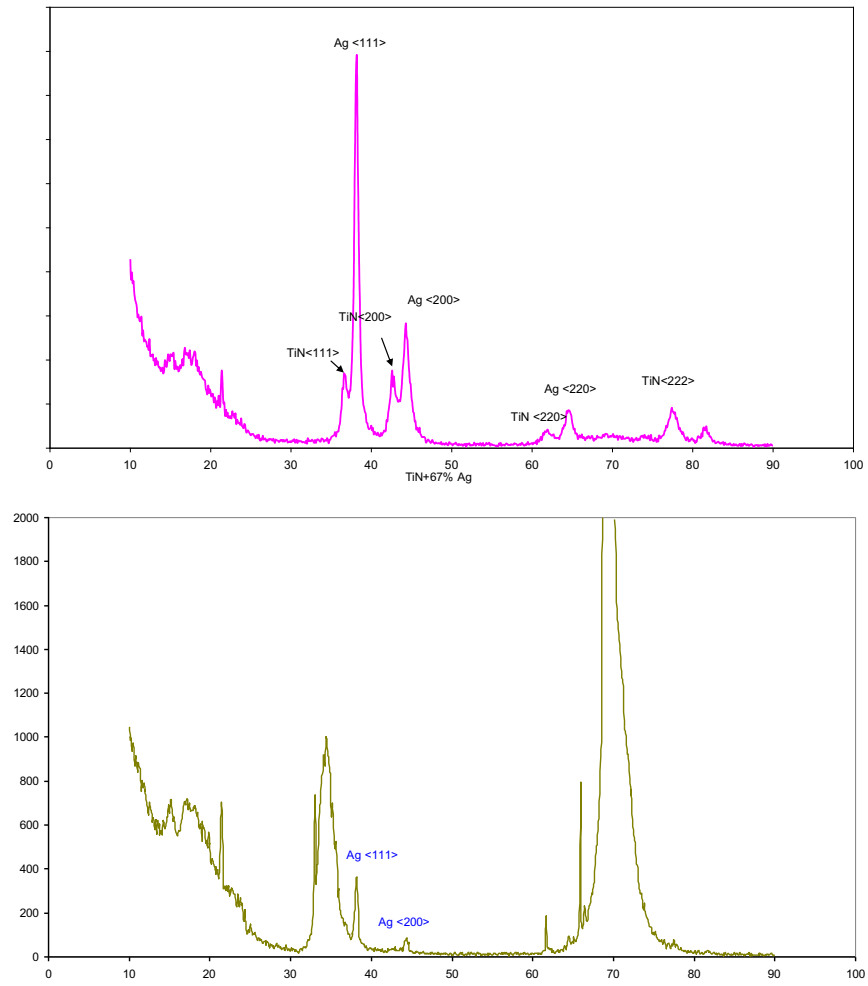

Fig. 6. XRD spectra coatings ref. TiN, TiN-20Ag, TiN-30Ag and TiN-66Ag (top to bottom). 
Table 1

Antimicrobial activity (R) and Ag release (mg/l) after 6 and $24 \mathrm{~h}$.

\begin{tabular}{lllll}
\hline & $\begin{array}{l}\text { Ag release }(6 \mathrm{~h}) \\
{[\mathrm{mg} / \mathrm{l}]}\end{array}$ & $\begin{array}{l}\mathrm{R}(6 \mathrm{~h}) \\
(\%)\end{array}$ & $\begin{array}{l}\text { Ag release }(24 \mathrm{~h}) \\
{[\mathrm{mg} / \mathrm{l}]}\end{array}$ & $\begin{array}{l}\mathrm{R}(24 \mathrm{~h}) \\
(\%)\end{array}$ \\
\hline $\begin{array}{c}\text { TiNAg }(50 \% \\
\mathrm{Ag})\end{array}$ & 0.08 & 99.4 & 0.15 & 99.7 \\
$\begin{array}{c}\mathrm{TiNAg}(25 \% \\
\mathrm{Ag})\end{array}$ & 0.07 & 91.3 & 0.21 & 97.5 \\
$\begin{array}{c}\text { TiNAg }(18 \% \\
\mathrm{Ag})\end{array}$ & $<0.06$ & 50 & 0.05 & 98.8 \\
$\begin{array}{c}\mathrm{TiNAg}(4 \% \\
\mathrm{Ag})\end{array}$ & $<0.05$ & 90.5 & 0.07 & 99.7 \\
\hline
\end{tabular}

\subsection{Coating Structure}

The incorporation of a metallic phase (Ag) into the TiN films could be expected to produce structural, morphological and chemical changes in the coating. [11] In fact, the silver amount in the coating had a remarkable consequence in the coating morphology (Fig. 5). Coating TiN-66Ag presented a coarse granular structure, which evolved towards a denser structure in lower silver content coatings: coating TiN-30Ag presented on average $1 \mu \mathrm{m}$ sized grains and coating TiN-20Ag $0.3 \mu \mathrm{m}$ ones.

The XRD spectra of coatings TiN-30Ag and TiN-20Ag showed the presence of two distinct phases, corresponding to $\mathrm{TiN}$ and $\mathrm{Ag}$ in the metallic face-centered cubic phase (FCC) (Fig. 6). The latter is due to the fact that silver is a noble metal and the metallic state is thus thermodynamically more stable than most silver compounds that can be produced by magnetron sputtering, i.e. the formation of silver nitrides is unlikely. On the other hand, only the metallic silver phase was identified on coating TiN-66Ag. This is in accordance with previous works that indicate that an increase in the silver content produces well-defined XRD diffraction patterns, evidencing an increment in the silver grain size when incorporated into crystalline MeN matrices. [11] The silver grain size increment is also accompanied by a decrease in the matrix grain size for crystalline matrices, i.e. TiN, caused by the hindrance of matrix grain growth due to silver segregation to the boundaries and/or the increased number of nucleation sites provided by silver particles/atoms. [11]

\subsection{Coating Antimicrobial Activity}

The antimicrobial activity of the coatings was assessed for references TiN-50Ag, TiN-25Ag, TiN-18Ag and TiN-04Ag (Table 1). In all tested coatings the reduction of the number of Staphylococcus epidermidis bacteria was above $97 \%$ after $24 \mathrm{~h}$, and already above $90 \%$ after $6 \mathrm{~h}$, except for ref. TiN-18Ag. This study showed again that the amount of silver on the surface cannot be regarded as the sole parameter controlling the antibacterial effect on ceramic-Ag films, as has been reported elsewhere. [11]

There is no agreement regarding how silver kills bacteria, but the main factors responsible for the antimicrobial capabilities of silver are directly related to the mechanisms controlling bacterial spreading, because $\mathrm{Ag}+$ interacts with the cell thiol groups by binding to the main functional groups in some components, such as enzymes, thus preventing bacterial division and damaging the cell envelope [30]. Therefore, we can hypothesize that the antimicrobial effect of the tested TiN-Ag coatings occurred both by direct coating-bacteria contact and by silver release into the culture media. However, in the latter case, there was not a direct correlation between the silver content on the coating and the amount of silver released, indication that the surface morphology also played a role.

Besides, in the case of the coatings with lower silver content, TiN$04 \mathrm{Ag}$ and TiN-18Ag, silver was rapidly released in the first six hours of immersion, reaching a plateau afterwards (Table 1). This might be linked to the formation of passive films on the outer surface of the coating, which prevents silver release. [31] In any case, these coatings
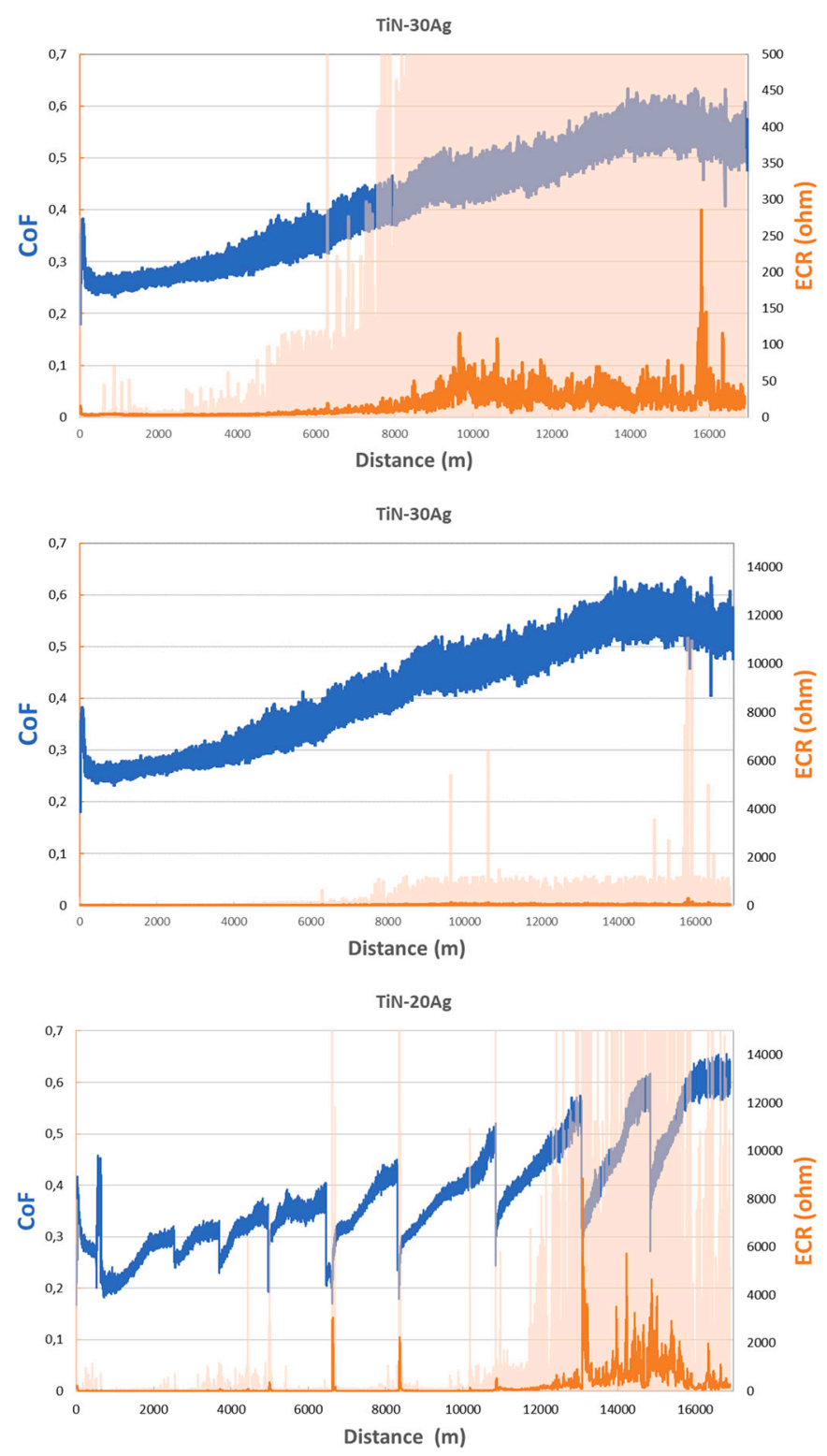

Fig. 7. Evolution of the Coefficient of Friction (COF; left vertical axis) and Electrical Contact Resistance (ECR: right vertical axis) of ref. TiN-30Ag (top and center, at different scales) and TiN-20Ag (below). NOTE: ECR test values are represented in light orange, and the average of 100 ECR test values in dark orange. (For interpretation of the references to colour in this figure legend, the reader is referred to the web version of this article.)

can be optimal for short-term antibacterial applications, e.g. surgical tools, because the silver release could prevent the possible bacterial adhesion while maintaining a low silver release, thus reducing undesired toxicity effects.

\subsection{Coating Wear Resistance}

Additionally to the antimicrobial activity, the tribology of many medical devices and surgical instruments plays often a key role. [32] Actually, heat produced at the tool tissue interface because of friction can produce thermal necrosis [33].

Silver might function as a solid lubricant on the surface of the material, reducing wear, provided silver is present or is segregated to the surface by the contact pressure or the temperature. [15] On the other hand, as have been exposed before, silver content decreases the coating hardness, which might have a deleterious effect on the wear resistance. 


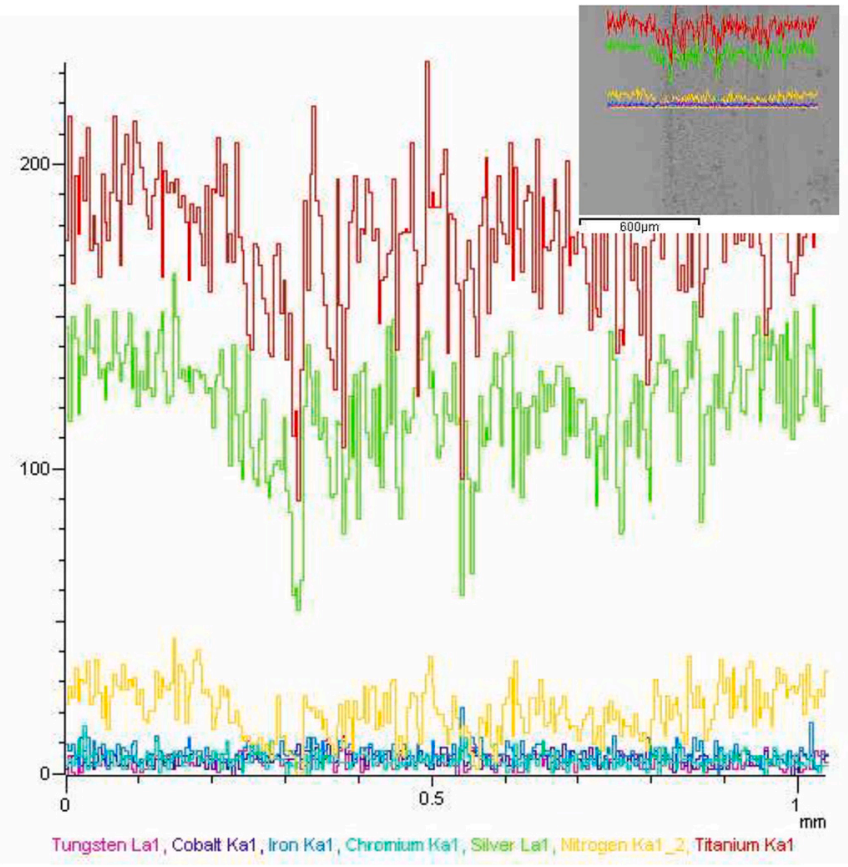

Fig. 8. Energy Dispersive Spectroscopy (EDS) profile of the wear track of the TiN-20Ag coating after the electro-tribology test (Scanning Electron Microscopy, SEM, image of the same wear track in the insertion).

\section{[29]}

In this work, the wear resistance of TiN-Ag was assessed against both WC-Co and synthetic bone material, at controlled humidity (50\%) and in dry conditions respectively.

In the first case, the TiN-20Ag and TiN-30Ag coatings were selected and applied on AISI 420A stainless steel discs. Along the tribological tests, the evolution of both the coefficient of friction (CoF) and the electrical contact resistance (ECR) were monitored. An increase of the $\mathrm{CoF}$ is typically deleterious in biomedical applications as it might increase the interface temperature and consequently damage the surrounding tissue. The ECR values provide information about the resistivity of the contacting materials, which can increase e.g. if oxides are formed along the tests, and/or the contact area, which is typically affected by wear in the form of surface roughening, presence of wear debris, adhesive material transfer, etc. The tested coatings showed different electro-tribological performances (Fig. 7). In the case of ref. TiN-30Ag, the CoF rose steadily from below 0.3 to values above 0.6 (Fig. 7, above). Oscillations in the CoF also increased along the test. In the case of the ECR initial readings were low, i.e. below $50 \Omega$, while CoF stayed below 0.3 . When the CoF rose above 0.4 the ERC also rose to values around $1000 \Omega$ (Fig. 7, middle). This indicates that the wear phenomenon was relatively mild in comparison to that of ref. TiN-20Ag.

Ref. TiN-20Ag showed overall a less steady performance associated to a stick-slip like phenomenon (Fig. 7, below). The CoF built up gradually, followed by a sudden drop to values below 0.2 . Eventually, the CoF built up to higher and higher values, from around 0.3 to above 0.6. This is linked to the adhesive nature of the wear along the test, i.e. some material transfer occurred from the coating to the ball counterpart, which eventually detached. These phenomena were accompanied by a sudden short time rise in the ECR value. However, in the later stages of the test, once the CoF rose above around 0.5, values of the ECR shot up above $10,000 \Omega$, indicating a more severe degradation of the surface. Despite this, the wear did not reach in any case the stainless steel substrate, as demonstrated by the EDS analyses of the wear track (Fig. 8). Significant amounts of surface oxides were not identified either. This indicates that the evolution of ECR was affected mainly by geometrical features defining the contact area.

As described above, the contrast in the behavior of surfaces TiN$20 \mathrm{Ag}$ and TiN-30Ag was stark. This might be due to a number of reasons. In the case of reference TiN-30Ag, a larger silver content might have produced a smoother transfer of material and deformation of the contact track, as the coating is relatively softer than TiN-20Ag, and thus avoidance of the stick-slip phenomena. The presence of two consecutive ECR value stages (Fig. 7, middle, in orange - right scale), points to the eventual stabilization ( $>$ around $8.000 \mathrm{~m}$ ) of the contact surface between the coating and the ball counterpart and the degree of surface oxidation.

On the other hand, ref. TiN-20Ag, with less metal content, presented a less cohesive material transfer, resulting, as the tension rose in the interacting surfaces, on the detachment of material. This might eventually have caused a roughening of the surfaces, enlargement of the contact areas and generation of oxidized layers to a lesser extent (below the detection limit of the EDS technique), and consequently a rise in ECR values.

Additionally, the TiN-04Ag coating was selected and applied on stainless steel manufactured reamers (Fig. 1). Uncoated reamers were also tested in the same condition. The worn areas at the cutting edges after the tests were quantified as $1.66 \mathrm{~mm}$ and $2.93 \mathrm{~mm}$ long for the TiN04Ag coated and uncoated reamers respectively (Fig. 9). Therefore, the coating almost halved the worn cutting-edge front, with a reduction of $43 \%$. Therefore, the cutting edge can keep the sharpness longer and potentially induce less damage on the living tissue counterpart.

\section{Conclusions}

The previous sections have shown how the silver content on TiN-Ag coatings depends on the deposition parameters, and how they affect the coating hardness and its adhesion to stainless steel. Great variations on the coating surface morphology have also been observed.
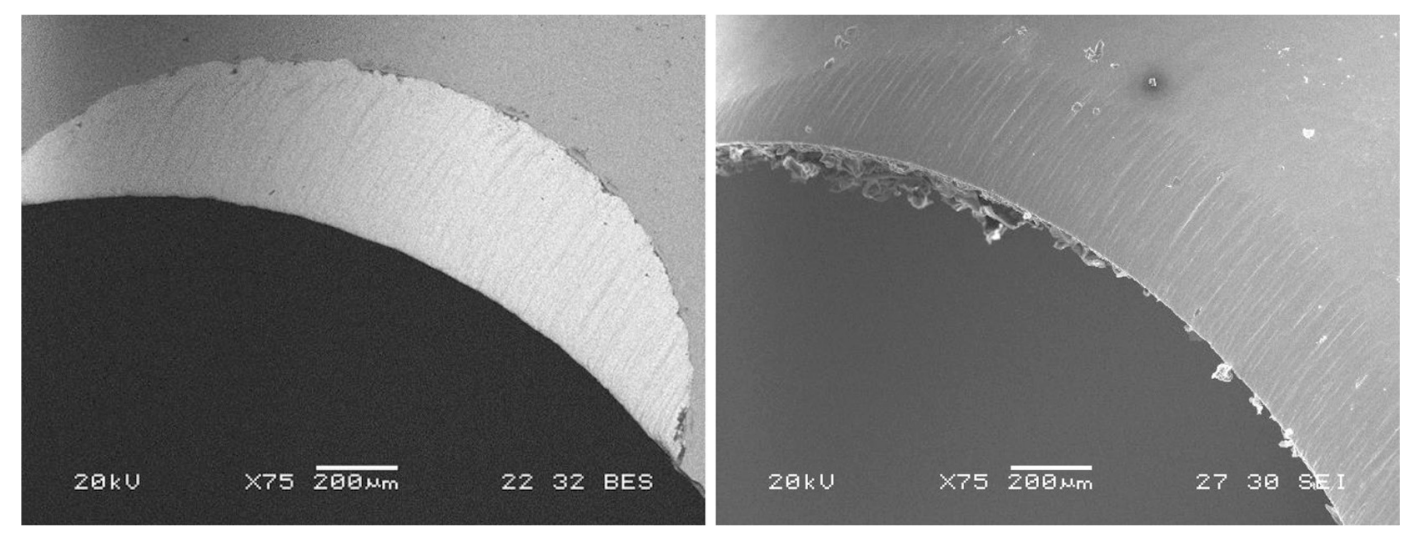

Fig. 9. SEM images of the of the cutting edges of TiN-04Ag coated (left) and uncoated (right) reamers. 
Both morphology and silver content have influenced the antimicrobial activity of the surface against Staphylococcus epidermidis, which is behind many of the more serious and problematic nosocomial hospital infections. In lower silver containing TiN-Ag coatings, additionally to the antimicrobial effect, it has been observed that a plateau of silver release is achieved in a short time, which can dispel any concern about potential toxicity effects and might be beneficial on surfaces that are in contact with living tissue for short periods of time, e.g. surgical tools.

Additionally, these low silver content TiN-Ag coatings provide a degree of wear resistance, thus minimizing wear debris and the associated concern about their likely toxicity and maximizing good quality tool cutting edge life that might reduce the necrosis induced in living tissue when applied on surgical tools. Finally, it has been found that the content of silver does change in a significant way the tribology performance, in terms of friction, of the coatings, and that monitoring the Electric Contract Resistance can provide a valuable insight about the status of this surfaces.

\section{Declaration of Competing Interest}

The authors declare that they have no known competing financial interests or personal relationships that could have appeared to influence the work reported in this paper.

\section{Acknowledgements}

This work was supported by the Economic Development Agency of the Basque Government under the following grants: Elkartek program, project Frontiers-V ref. KK-2019/00077 and BACTERICOAT project N. EXPT IG-2006/0000465, IT-2007/0000623, IT-2008/0000488.

\section{References}

[1] D. Campoccia, L. Montanaro, C. Renata Arciola, The significance of infection related to orthopedic devices and issues of antibiotic resistance, Biomaterials 27 (2006) 2331-2339, https://doi.org/10.1016/j.biomaterials.2005.11.044.

[2] C.L. Romanò, H. Tsuchiya, I. Morelli, A.G. Battaglia, L. Drago, Antibacterial coating of implants: are we missing something? Bone Joint Res. 8 (2019) 199-206, https:// doi.org/10.1302/2046-3758.85.BJR-2018-0316.

[3] T.F. Moriarty, R. Kuehl, T. Coenye, W.-J. Metsemakers, M. Morgenstern, E. M. Schwarz, M. Riool, S.A.J. Zaat, N. Khana, S.L. Kates, R.G. Richards, Orthopaedic device-related infection: current and future interventions for improved prevention and treatment, Effort Open Rev. 1 (2016) 89-99, https://doi.org/10.1302/20585241.1 .000037$.

[4] A. Trampuz, W. Zimmerli, Diagnosis and treatment of infections associated with fracture-fixation devices, Injury 37 (2006) S59-S66, https://doi.org/10.1016/j. injury.2006.04.010.

[5] J.D. Davidson, D. Spratt, A.D. Liddle, Implant materials and prosthetic joint infection: the battle with the biofilm, Effort Open Rev. 4 (2019) 633-639, https:// doi.org/10.1302/2058-5241.4.180095.

[6] F.M. Gomes Pinto, R. Queiroz de Souza, C. Barreto da Silva, L.M. Jenné Mimica, K. Uchikawa Graziano, Analysis of the microbial load in instruments used in orthopedic surgeries, Am. J. Infect. Control 38 (3) (2010) 229-233, https://doi. org/10.1016/j.ajic. 2009.06.017.

[7] I. Braceras, P. Azpiroz, N. Briz, R.M. Fratila, J. Oyarbide, E. Ipinazar, N. Álvarez, G. Atorrasagasti, J.M. Aizpurua, Plasma polymerized Silylated ciprofloxacin as an antibiotic coating, Plasma Process. Polym. 8 (7) (2011) 599-606, https://doi.org/ 10.1002/ppap. 201190013.

[8] I. Braceras, J. Oyarbide, P. Azpiroz, N. Briz, E. Ipiñazar, N. Álvarez, G. Atorrasagasti, R.M. Fratila, J.M. Aizpurua, "Plasma-click" based strategy for obtaining antibacterial surfaces on implants, Plasma Process. Polym. 10 (4) (2013) 328-335, https://doi.org/10.1002/ppap.201200058.

[9] M. Cloutier, D. Mantovani, F. Rosei, Antibacterial coatings: challenges, perspectives, and opportunities, Trends Biotechnol. 33 (11) (2015) 637-652, https://doi.org/10.1016/j.tibtech.2015.09.002.

[10] J. Zhao, X.M. Cai, H.Q. Tang, T. Liu, H.Q. Gu, R.Z. Cui, Bactericidal and biocompatible properties of TiN/Ag multilayered films by ion beam assisted deposition, J. Mater. Sci. Mater. Med. 20 (2009) S101-S105, https://doi.org/ 10.1007/s10856-008-3491-5.
[11] S. Calderon Velasco, A. Cavaleiro, S. Carvalho, Functional properties of ceramic-Ag nanocomposite coatings produced by magnetron sputtering, Prog. Mater. Sci. 84 (2016) 158-191, https://doi.org/10.1016/j.pmatsci.2016.09.005.

[12] O.M. Goudouri, E. Kontonasaki, U. Lohbauer, A.R. Boccaccini, Antibacterial properties of metal and metalloid ions in chronic periodontitis and peri-implantitis therapy, Acta Biomater. 10 (8) (2014) 3795-3810, https://doi.org/10.1016/j. actbio.2014.03.028

[13] P.J. Kelly, H. Li, K.A. Whitehead, J. Verran, R.D. Arnell, I. Iordanova, A study of the antimicrobial and tribological properties of TiN/Ag nanocomposite coatings, Surf. Coat. Technol. 204 (2009) 1137-1140, https://doi.org/10.1016/j. surfcoat.2009.05.012.

[14] P.J. Kelly, H. Li, P.S. Benson, K.A. Whitehead, J. Verran, R.D. Arnell, I. Iordanova, Comparison of the tribological and antimicrobial properties of $\mathrm{CrN} / \mathrm{Ag}, \mathrm{ZrN} / \mathrm{Ag}$, TiN/Ag, and TiN/cu nanocomposite coatings, Surf. Coat. Technol. 205 (2010) 1606-1610, https://doi.org/10.1016/j.surfcoat.2010.07.029.

[15] H. Ju, L. Yu, D. Yu, I. Asempah, J. Xu, Microstructure, mechanical and tribological properties of TiN-Ag films deposited by reactive magnetron sputtering, Vacuum 141 (2017) 82-88, https://doi.org/10.1016/j.vacuum.2017.03.026.

[16] M. Zhao, H. Gong, M. Ma, L. Dong, M. Huang, R. Wan, H. Gu, Y. Kang, D. Li, A comparative antibacterial activity and cytocompatibility for different top layers of TiN, Ag or TiN-Ag on nanoscale TiN/Ag multilayers, Appl. Surf. Sci. 473 (2019) 334-342, https://doi.org/10.1016/j.apsusc.2018.12.159.

[17] J.L. Clement, P.S. Harrett, Antibacterial silver, Metal-Based Drugs 1 (1994) 467-482, https://doi.org/10.1155/MBD.1994.467.

[18] J.M. Schierholz, L.J. Lucas, A. Rump, G. Pulverer, Efficacy of silver-coated medical devices, J. Hosp. Infect. 40 (1998) 257-262, https://doi.org/10.1016/s0195-6701 (98)90301-2.

[19] D. Troitzsch, U. Borutzky, U. Junghannß, Detection of antimicrobial efficacy in silver-coated medical devices, Hyg. Med. 34 (3) (2009) 80-85.

[20] S.L. Percival, W. Slone, S. Linton, T. Okel, L. Corum, J.G. Thomas, The antimicrobial efficacy of a silver alginate dressing against a broad spectrum of clinically relevant wound isolates, Int. Wound J. 8 (2011) 237-243, https://doi. org/10.1111/j.1742-481X.2011.00774.x.

[21] A.J. Tande, R. Patel, Prosthetic joint infection, Clin. Microbiol. Rev. 27 (2014) 302-345, https://doi.org/10.1128/CMR.00111-13.

[22] C.R. Arciola, D. Campoccia, L. Montanaro, Implant infections: adhesion, biofilm formation and immune evasion, Nat. Rev. Microbiol. 16 (2018) 397-409, https:// doi.org/10.1038/s41579-018-0019-y.

[23] I. Braceras, I. Ibáñez, M. Taher, F. Mao, A. del Barrio, S. Saenz De Urturi, P. Berastegui, A.M. Andersson, Ulf Jansson, On the electro-tribological properties and degradation resistance of silver-aluminum coatings, Wear 414-415 (2018) 202-211, https://doi.org/10.1016/j.wear.2018.08.014.

[24] F.G. Echeverrigaray, S.R.S. de Mello, L.M. Leidens, C.D. Boeira, A.F. Michels, I. Braceras, C.A. Figueroa, Electrical contact resistance and tribological behaviors of self-lubricated dielectric coating under different conditions, Tribol. Int. 143 (2020) 106086, https://doi.org/10.1016/j.triboint.2019.106086.

[25] W.F. Sherman, T.R. Flick, C.S. Dranoff, M.J. Weintraub, N.N. Kale, C. Sommi, F. L. Sanchez, Variability of cutting and thermal dynamics between new and used acetabular reamers during total hip arthroplasty, J. Mech. Behav. Biomed. Mater. 3 (2010) 549-558, https://doi.org/10.1016/j.artd.2020.12.002.

[26] S. Slotkin, N.B. Frisch, G. Roc, C.D. Silverton, Hemispherical and minimally invasive total hip reamers: a biomechanical analysis of use and design, Arthroplasty Today 3 (2017) 131-136, https://doi.org/10.1016/j. artd.2016.09.009.

[27] V.S. Dhandapani, E. Thangavel, M. Arumugam, K.S. Shin, V. Veeraraghavan, S. Y. Yau, C. Kim, D.-E. Kim, Effect of Ag content on the microstructure, tribological and corrosion properties of amorphous carbon coatings on 316L SS, Surf. Coat. Technol. 240 (2014) 128-136, https://doi.org/10.1016/j.surfcoat.2013.12.025.

[28] V.S. Calderon, J.C. Oliveira, M. Evaristo, A. Cavaleiro, S. Carvalho, Prediction of optimized composition for enhanced mechanical and electrochemical response of Zr-C-N-Ag coatings for medical devices, Appl. Surf. Sci. 320 (2014) 570-580, https://doi.org/10.1016/j.apsusc.2014.08.201.

[29] H. Köstenbauer, G. Fontalvo, C. Mitterer, J. Keckes, Tribological properties of TiN/ Ag nanocomposite coatings, Tribol. Lett. 30 (2008) 53-60, https://doi.org/ 10.1007/s11249-008-9312-7.

[30] W.K. Jung, H.C. Koo, K.W. Kim, S. Shin, S.H. Kim, Y.H. Park, Antibacterial activity and mechanism of action of the silver ion in Staphylococcus aureus and Escherichia coli, Appl. Environ. Microbiol. 74 (2008) 2171-2178, https://doi.org/10.1128/ AEM.02001-07.

[31] S. Rtimi, O. Baghriche, R. Sanjines, C. Pulgarin, M. Ben-Simon, J.C. Lavanchy, A. Houas, J. Kiwi, Photocatalysis/catalysis by innovative TiN and TiN-Ag surfaces inactivate bacteria under visible light, Appl. Catal. B 123-124 (2012) 306-315, https://doi.org/10.1016/j.apcatb.2012.04.047.

[32] Z.M. Jin, J. Zheng, W. Li, Z.R. Zhou, Tribology of medical devices, Biosurf. Biotribol. 2 (2016) 173-192, https://doi.org/10.1016/j.bsbt.2016.12.001.

[33] G. Augustin, T. Zigman, S. Davila, T. Udilljak, T. Staroveski, D. Brezak, S. Babic, Cortical bone drilling and thermal osteonecrosis, Clin. Biomech. 27 (2012) 313-325, https://doi.org/10.1016/j.clinbiomech.2011.10.010. 